\title{
Quantitative proteomics and transcriptomics of anaerobic and aerobic yeast cultures reveals post- transcriptional regulation of key cellular processes
}

Correspondence

Monique Slijper

m.slijper@uu.nl
Received 22 May 2007

Revised 24 July 2007

Accepted 26 July 2007
Marco J. L. de Groot, ${ }^{1,4,5}+\ddagger$ Pascale Daran-Lapujade, ${ }^{2,4} \uparrow$ Bas van Breukelen, ${ }^{1,5}$ Theo A. Knijnenburg,, ${ }^{3,4}$ Erik A. F. de Hulster, ${ }^{2,4}$ Marcel J. T. Reinders, ${ }^{3,4}$ Jack T. Pronk, ${ }^{2,4}$ Albert J. R. Heck ${ }^{1,5}$ and Monique Slijper ${ }^{1,5}$

${ }^{1}$ Department of Biomolecular Mass Spectrometry, Bijvoet Center for Biomolecular Research and Utrecht Institute for Pharmaceutical Sciences, Utrecht University, Sorbonnelaan 16, 3584 CA Utrecht, The Netherlands

${ }^{2}$ Department of Biotechnology, Delft University of Technology, Julianalaan 67, 2628 BC Delft, The Netherlands

${ }^{3}$ Information and Communication Theory Group, Faculty of Electrical Engineering, Mathematics and Computer Science, Delft University of Technology, Mekelweg 4, 2628 CD Delft, The Netherlands

${ }^{4}$ Kluyver Centre for Genomics of Industrial Fermentation, Delft, The Netherlands

${ }^{5}$ Netherlands Proteomics Centre, Utrecht, The Netherlands

Saccharomyces cerevisiae is unique among yeasts in its ability to grow rapidly in the complete absence of oxygen. S. cerevisiae is therefore an ideal eukaryotic model to study physiological adaptation to anaerobiosis. Recent transcriptome analyses have identified hundreds of genes that are transcriptionally regulated by oxygen availability but the relevance of this cellular response has not been systematically investigated at the key control level of the proteome. Therefore, the proteomic response of $S$. cerevisiae to anaerobiosis was investigated using metabolic stable-isotope labelling in aerobic and anaerobic glucose-limited chemostat cultures, followed by relative quantification of protein expression. Using independent replicate cultures and stringent statistical filtering, a robust dataset of 474 quantified proteins was generated, of which 249 showed differential expression levels. While some of these changes were consistent with previous transcriptome studies, many of the responses of $S$. cerevisiae to oxygen availability were, to our knowledge, previously unreported. Comparison of transcriptomes and proteomes from identical cultivations yielded strong evidence for post-transcriptional regulation of key cellular processes, including glycolysis, amino-acyl-tRNA synthesis, purine nucleotide synthesis and amino acid biosynthesis. The use of chemostat cultures provided well-controlled and reproducible culture conditions, which are essential for generating robust datasets at different cellular information levels. Integration of transcriptome and proteome data led to new insights into the physiology of anaerobically growing yeast that would not have been apparent from differential analyses at either the mRNA or protein level alone, thus illustrating the power of multi-level studies in yeast systems biology.

†These authors contributed equally to this work

łPresent address: Information and Communication Theory Group, Delft University of Technology, 2628 CD Delft, The Netherlands.

Abbreviations: aaRS, aminoacyl-tRNA synthetase; ESI, electrospray ionization; LC, liquid chromatography; MIPS, Munich Information Center for Protein Sequences; PCA, principal component analysis; PDF, probability density function; $\mathrm{R}_{\mathrm{Anae}}$ : Ae, relative protein ratios (anaerobic : aerobic); RMS, root-meansquare; TCA cycle, tricarboxylic acid cycle.

Data from this study have been stored at http://www.ncbi.nlm.nih.gov/geo/, as follows: description of the proteins identified, platform GPL4992; description of the biological material, table of quantitative protein data and link to XLS file containing peptide data, sample GSM177360; summary of the overall experiment and table of mRNA comparison data, series GSE7365.

A supplementary figure and two supplementary tables are available with the online version of this paper. 


\section{INTRODUCTION}

The yeast Saccharomyces cerevisiae stands out among yeast species for its rapid growth in the complete absence of oxygen (Visser et al., 1990). This makes S. cerevisiae an ideal eukaryotic model to study physiological adaptation to anaerobiosis. Besides this fundamental interest, many industrial applications of $S$. cerevisiae, such as the leavening of bread, beer brewing and bioethanol production, occur under essentially anaerobic conditions.

The unique ability of $S$. cerevisiae to grow both aerobically and anaerobically has been investigated for decades through physiological and genetic studies. When cells are grown aerobically, molecular oxygen serves as the final electron acceptor for respiration, while it is also used in haemoprotein synthesis and in synthesis of metabolites, e.g. sterols, unsaturated fatty acids and nicotinamide, which are involved in specific anabolic pathways (Rosenfeld \& Beauvoit, 2003). In the absence of oxygen, respiration can no longer occur, and $S$. cerevisiae becomes auxotrophic for specific compounds. Metabolism is then reprogrammed to optimize yeast cells for fermentative dissimilation of the carbon source in order to conserve energy and maintain a closed redox balance. These metabolic rearrangements are easily detectable by analysis of metabolites, such as glycerol and ethanol, in the culture supernatant. The effect of the absence of oxygen on yeast has more recently been investigated under steady-state and dynamic conditions by genome-wide transcriptome analyses (Kwast et al., 2002; Lai et al., 2005; Piper et al., 2002; Salusjarvi et al., 2003; ter Linde et al., 1999). Hundreds of genes appeared to be transcriptionally regulated by oxygen availability; these changes in transcriptional regulation go well beyond the changes required to switch between respiratory and fermentative metabolism and highlight the complex nature of anaerobic growth. Although the analysis of mRNA has proven to be very powerful in the discovery of molecular markers and the elucidation of functional mechanisms, it is not sufficient for full characterization of biological systems. Several previous studies have shown that transcript levels are imperfect indicators of protein levels and in vivo fluxes (Daran-Lapujade et al., 2004; Griffin et al., 2002; Kolkman et al., 2006; Washburn et al., 2003a). Studies at the level of protein activity have been performed by in vivo enzyme assays, but, due to the tediousness of such assays, they have been restricted to small numbers of proteins (Machado et al., 1975; Servouse \& Karst, 1986; van Hoek et al., 2000). To date, a detailed quantitative analysis of yeast proteome changes in response to oxygen availability is still lacking.

For a systems-biology approach, our aim was firstly to obtain a quantitative proteome dataset, and secondly to compare the changes in protein expression levels with changes in mRNA measured previously by Tai et al. (2005). Such quantitative approaches require thorough experimental design of both the analytical and the cultivation techniques. Laboratory-scale cultivation of micro-organisms is predominantly performed in shake flasks; during this process the physical and chemical environment constantly changes, which affects the specific growth rate and the regulation of many metabolic processes. As the time constants of transcription and translation, and also the turnover rates of mRNAs and their corresponding proteins are likely to differ, the dynamic nature of batch cultures complicates studies of correlations between mRNA and protein levels. The use of a chemostat offers the unique possibility of studying metabolism and its regulation, allowing a fixed and constant specific growth rate under tightly defined nutritional conditions. Therefore chemostats are excellent tools for accurate quantitative transcriptome and proteome studies (Daran-Lapujade et al., 2004; Kolkman et al., 2005; Tai et al., 2005).

In the present work the remodelling of the $S$. cerevisiae proteome elicited by anaerobiosis was investigated in steady-state in glucose-limited chemostat cultures by metabolic stable-isotope labelling. Yeast proteins were labelled in chemostat cultures using growth media with either ${ }^{14} \mathrm{~N}$ or ${ }^{15} \mathrm{~N}$ as the single nitrogen source. Proteins were subsequently separated by $1 \mathrm{D}$ gel electrophoresis and reversed-phase liquid chromatography (RPLC), and analysed by mass spectrometry to determine relative protein levels. Using independent culture replicates and stringent statistical filtering, we defined a robust dataset of 474 quantified proteins. In addition to the identification of changes in protein abundance that could be expected from current knowledge, this dataset revealed many hitherto unreported responses of S. cerevisiae to oxygen availability. Furthermore, the reliable quantification of changes in protein expression and their comparison with genomewide transcript levels from scrupulously identical cultures enabled the discovery of post-transcriptional regulation. The clustering of protein and mRNA expression data by functional categories suggested that these regulatory mechanisms function at the level of whole pathways (glycolysis and purine and amino acid synthesis) and for entire groups of proteins sharing similar cellular functions (aminoacyl-tRNA synthetases).

\section{METHODS}

Overview of experimental setup. The relative protein levels of anaerobically versus aerobically grown $S$. cerevisiae were investigated using metabolic stable-isotope labelling. The biomass was produced using carbon-limited chemostat cultures. Each biomass was produced twice, once using a ${ }^{14} \mathrm{~N}$-labelled nitrogen source (ammonium sulfate) and once using a $98 \%{ }^{15} \mathrm{~N}$-labelled nitrogen source. Subsequently, each biomass was harvested, and protein extracts were prepared. Two mixed samples were prepared from the four protein samples, containing equal quantities of protein from ${ }^{14} \mathrm{~N}$ aerobic and ${ }^{15} \mathrm{~N}$ anaerobic cultures for the first experiment and equal quantities of protein from ${ }^{15} \mathrm{~N}$ aerobic and ${ }^{14} \mathrm{~N}$ anaerobic cultures for the second experiment. Proteins of the two mixed samples were separated via SDS-PAGE, and each lane was equally fragmented into 40 slices, resulting in subsamples based on protein size. This was followed by in-gel protein digestion using trypsin, and analysis in triplicate by nanoflow-liquid chromatography (LC)-MS/MS. Proteins were identified using MS/MS, and the relative protein levels were obtained by 
comparing the extracted ion chromatograms of the co-eluting ${ }^{14} \mathrm{~N}$ and ${ }^{15} \mathrm{~N}$-labelled peptide pairs.

Strain and growth conditions. The haploid, prototrophic Saccharomyces cerevisiae strain CEN.PK113-7D (MATa, MAL2-8, SUC2, obtained from P. Kötter, Institut für Mikrobiologie, J. W. Goethe Universität Frankfurt, Frankfurt, Germany) was grown at $30{ }^{\circ} \mathrm{C}$ in 21 chemostats (Applikon), with a working volume of $1.0 \mathrm{l}$ as described by Verduyn et al. (1992). Cultures were fed with a defined mineral medium that limited growth by glucose and supplied all other growth requirements in excess. The dilution rate (equivalent to growth rate in steady-state cultures) was set at $0.10 \mathrm{~h}^{-1}$. The $\mathrm{pH}$ was measured online and kept constant at 5.0 by the automatic addition of $2 \mathrm{M} \mathrm{KOH}$ with the use of an Applikon ADI 1030 biocontroller. Stirrer speed was maintained at 800 r.p.m. and the gas inflow was $0.51 \mathrm{~min}^{-1}$. Aerobic cultures were sparged with air; the dissolved oxygen tension was measured online with an oxygen electrode (Ingold model 341003002 ), and was above $60 \%$ of air saturation. For anaerobic chemostat cultivations, fermenters were equipped with Norprene tubing and O-rings to avoid oxygen diffusion, and both the fermenter and medium vessel were continuously sparged with ultrapure nitrogen gas containing less than 5 p.p.m. oxygen (Hoekloos), as described by Visser et al. (1990). The off-gas was cooled by a condenser connected to a cryostat set at $2{ }^{\circ} \mathrm{C}$ and analysed as previously described (van Maris et al., 2003). Steady-state samples were taken after approximately 10-14 volume changes to avoid strain adaptation due to long-term cultivation (Jansen et al., 2005). Biomass dry-weight, metabolite, dissolved oxygen and gas profiles were constant over at least five volume changes prior to sampling for analysis.

Media. The defined synthetic mineral medium composition was based on that described by Verduyn et al. (1992); the $\left(\mathrm{NH}_{4}\right)_{2} \mathrm{SO}_{4}$ concentration was, however, changed to $19 \mathrm{mM}$. The aerobic cultures were fed with $\sim 7.5 \mathrm{~g}$ glucose $1^{-1}$ and the anaerobic cultures with $\sim 25 \mathrm{~g}$ glucose $1^{-1}$. For anaerobic cultivations, the medium was supplemented with the anaerobic growth factors Tween-80 and ergosterol (420 and $10 \mathrm{mg} \mathrm{l}^{-1}$, respectively) (Verduyn et al., 1990).

Analytical methods. Culture supernatants were obtained after centrifugation of chemostat samples at $5000 \mathrm{~g}$. HPLC analysis was performed to analyse both these culture supernatant samples and the culture media. The chromatograph was equipped with an AMINEX HPX-87H ion exchange column; $5 \mathrm{mM} \mathrm{H}_{2} \mathrm{SO}_{4}$ was used as the mobile phase. Culture dry weights were determined via filtration as described by Postma et al. (1989). Protein content of yeast biomass was analysed by a modified biuret method (Verduyn et al., 1990)

Sample preparation. Samples for proteome analysis were kept on ice and immediately centrifuged $\left(12000 \mathrm{~g}, 5 \mathrm{~min}\right.$ at $\left.4{ }^{\circ} \mathrm{C}\right)$, washed twice with ice-cold water, and stored $5 \times$ concentrated in water at $-80{ }^{\circ} \mathrm{C}$. Yeast cells were lyophilized prior to protein extraction. For protein extraction, a $25 \mathrm{mg}$ cellular dry-weight sample was mixed with $750 \mu$ twofold-concentrated SDS loading buffer [60 mM Tris/ $\mathrm{HCl}, \mathrm{pH} 6.8,2.5 \%(\mathrm{w} / \mathrm{v})$ SDS, $10 \%(\mathrm{v} / \mathrm{v})$ glycerol and protease inhibitors (Complete, Protease Inhibitor Cocktail tablets; Roche Diagnostics)]. Glass beads (Sigma, acid-washed, 425-600 $\mu \mathrm{m}$ diameter) were added and the cells were disrupted by vortexing five times $\left(60 \mathrm{~s}\right.$ at $\left.4{ }^{\circ} \mathrm{C}\right)$ in alternation with cooling intervals ( $30 \mathrm{~s}$ on ice). The supernatant from the beads was collected after mild centrifugation at $5000 \mathrm{~g}$ for $1 \mathrm{~min}$. This extraction step was repeated for the pelleted cells, after which both supernatants were pooled. Lysates, containing mostly the soluble proteins, were further cleared by centrifugation at $3000 \mathrm{~g}$ for $10 \mathrm{~min}$ at $4{ }^{\circ} \mathrm{C}$. Protein concentrations were determined using the 2D Quant kit (GE Healthcare). The protein extracts were stored in aliquots of $125 \mu \mathrm{g}$ at $-80{ }^{\circ} \mathrm{C}$.
Protein separation and in-gel tryptic digestion. DTT was added to each $125 \mu \mathrm{g}$ protein sample at a final concentration of $100 \mathrm{mM}$, after which the sample was boiled for 5 min prior to loading onto a gel. Equal amounts of protein from each sample (of the ${ }^{14} \mathrm{~N}$-labelled and of the ${ }^{15} \mathrm{~N}$-labelled culture) were mixed and $250 \mu \mathrm{g}$ of the protein mixture was separated through electrophoresis on a home-made, $13 \mathrm{~cm}$ long, $12 \%$ SDS-PAGE gel. Proteins were visualized using Gelcode Blue Stain Reagent (Pierce). The gel lane was cut into 40 slices, each slice was cut into small pieces. The gel pieces were further processed as described previously (Kolkman et al., 2006).

Nanoflow-LC-MS/MS. Nanoflow-LC-MS/MS analysis was performed by coupling an Agilent 1100 Series LC system (vacuum degasser, auto sampler and one high-pressure-mixing binary pump without static mixer) to an LCQ Classic quadrupole ion trap mass spectrometer (Finnigan). Peptides were delivered to a trap column [Aqua C18RP (Phenomenex), $15 \mathrm{~mm} \times 100 \mu \mathrm{m}$ internal diameter, packed in-house] at $5 \mu \mathrm{lmin}^{-1} 100 \%$ buffer A (0.1 M acetic acid). After reducing the flow to approximately $150 \mathrm{nl} \mathrm{min}{ }^{-1}$ using a splitter, the peptides were eluted over the analytical column [Aqua C18RP (Phenomenex), $15 \mathrm{~cm} \times 75 \mu \mathrm{m}$ internal diameter, packed in-house] with a linear gradient from 0 to $50 \%$ buffer $\mathrm{B}(0.1 \mathrm{M}$ acetic acid in $80 \%, \mathrm{v} / \mathrm{v}$, acetonitrile) in $120 \mathrm{~min}$. The column eluent was sprayed directly into the electorospray ionization (ESI) source of the mass spectrometer via a butt-connected nano-ESI emitter (New Objectives). The LCQ operated in positive-ion mode, and peptides were fragmented in data-dependent mode. One MS survey scan was followed by one data-dependent MS/MS scan (adapted from Meiring et al., 2002). Each digest was analysed in triplicate using nanoflow-LC-MS/MS.

Protein identification. The SEQUEST algorithm (Eng et al., 1994) was used to interpret the MS/MS spectra against the complete nonredundant proteome database of $S$. cerevisiae in FASTA format, obtained from the European Bioinformatics Institute (EBI) (Washburn et al., 2002, 2003a, b). The algorithm was run twice on each raw data file, firstly identifying proteins from the peptides containing ${ }^{14} \mathrm{~N}$, and secondly those from peptides containing ${ }^{15} \mathrm{~N}$ as described by Kolkman et al. (2006). The false positive rate of peptide identifications was determined by using the same database with all the amino acid sequences reversed (Krijgsveld et al., 2006).

Relative quantification of the proteins. Ion chromatograms from each peptide, selected using DTAselect, were extracted from the Xcalibur raw data file by a modified version of EXTRACT-CHRO. The relative protein levels were determined by calculating peptide ion current ratios using RelEx software (MacCoss et al., 2003), which were also inspected manually. A number of proteins were solely detected in yeast samples from one growth condition; we defined these proteins and their peptides as 'on-off. The corresponding extracted ion chromatograms of these 'on-off' peptides were filtered from the dataset. The remaining peptide ratios were calculated by using the following settings: peakshift $=1$; scans before and after $=2$; threshold factor $=0.55$; Savitsky-Golay filter $=7$ points; minimum correlations at 1 and 10 are 0.75 and 0.85 respectively; $\mathrm{S} / \mathrm{N}$ filter $=3$. Peptide ratio outliers were removed and the relative protein ratios (anaerobic:aerobic) were calculated by the RelEx software. These protein ratios were manually corrected when the average deviation was more than $10 \%$, based on correct peak detection or correlation within RelEx.

Data analysis. Principal component analysis (PCA) and hypergeometric testing were applied to determine which types of yeast proteins were preferentially detected by the analytical method used. PCA was performed using multivariate analysis in MiniTAB v14 (http://www. minitab.com/). 'Functional categories' and 'subcellular localizations' were as defined at the MIPS website (http://mips.gsf.de/). A particular 'functional category' or a 'subcellular localization' protein-expression 
group was only considered for further analysis if it contained five or more identified proteins. Significance of over-representation within the 'functional categories' of the different protein expression groups was determined using a hypergeometric test (Barash et al., 2001). Similarly, the representation of the identified proteins was analysed according to their sublocalization in the yeast cell.

Gene expression data. Systematic analysis of the control levels at which the yeast response to anaerobiosis takes place was performed using previously published transcript data obtained from yeast cultures grown under strictly identical conditions as described for the current proteome analysis. Affymetrix microarrays from five aerobic and four anaerobic independent culture replicates were used for this analysis. These array data are publicly available at the gene expression repository Gene Expression Omnibus (http:// www.ncbi.nlm.nih.gov/geo/) under accession number GSE4807.

Probability density test. A quantitative comparison between the protein anaerobic:aerobic ratios and the mRNA anaerobic:aerobic ratios was made. Of the 474 quantified proteins, 56 proteins were solely detected in a sample from one culture condition. Consequently, these genes cannot be assigned a protein ratio. Another 14 genes were not present on the microarray employed, and could thus not be assigned an mRNA ratio. As a result, transcriptome and proteome ratios could be obtained for 404 genes. The anaerobic: aerobic ratios of these 404 genes can be represented in a two-dimensional space, i.e. for each gene we have an $x$-coordinate, its mRNA ratio, and a $y$ coordinate, its protein ratio. A test has been designed to assess whether the genes from a particular MIPS functional category occupy a specific space in the distribution of all 404 genes. In this test, the following null hypothesis was applied:

$\mathrm{H}_{0}$ : The genes (data points) corresponding to a particular MIPS functional category are randomly sampled from all genes.

For example, if 10 of the 404 genes belong to a MIPS category, the null hypothesis assumes that each of these 10 genes could be any of the 404 points with equal probability, and thus, there is no specific location in this two-dimensional space where these 10 points are concentrated.

To test this hypothesis, the probability density function (PDF) of the complete distribution was compared with the PDF of a particular MIPS category. The non-parametric Parzen estimator using Gaussian kernels was applied to estimate the PDFs. Here, the smoothing parameter (kernel width, or in this case the variance of the Gaussian distribution) was optimized using the leave-one-out technique as described by Duin (1976). Basically, this density estimator models each point in the twodimensional space as a (Gaussian) bell-shaped distribution, where the height of the bell is perpendicular to the $2 \mathrm{D}$ space. For points that are close to each other, these bell-shaped distributions will overlap and thus add up. In this way a density estimate for the distribution of points is obtained. The PDFs are evaluated at a grid of 2500 uniformly spaced co-ordinates in the space spanned by the range of the mRNA and protein ratios (i.e. $-5.5<x<3$ and $-4<y<4$ ). In other words, the density estimate is computed for each of the 2500 grid coordinates. The sum of probabilities over all grid coordinates was set to one. The RMS (root-mean-square) value of the difference between the PDF estimated using the complete set of points $\left(P^{\text {all }}\right)$ and the PDF estimated using the points corresponding to a particular MIPS category $\left(P^{\mathrm{MIPS}}\right)$ is computed as follows:

$\mathrm{RMS}=\sqrt{\frac{1}{2500} \sum_{i=1}^{2500}\left(P_{i}^{\text {all }}-P_{i}^{\mathrm{MIPS}}\right)^{2}}$

where $P_{i}^{\text {all }}$ and $P_{i}^{\mathrm{MIPS}}$ represent the values of the estimated PDF of all genes or of the genes within the specified MIPS category, respectively, evaluated for the $i$-th grid coordinate. Here, the rationale is that if the null hypothesis $\mathrm{H}_{0}$ is true, i.e. the points that correspond to a MIPS category are randomly drawn from all points, the two PDFs will be very similar and hence the RMS will be very small. If the points of a MIPS category are located in a specific space within the cloud of points, there will be a large difference between the two PDFs and consequently a large value of the RMS score. Fig. 4 displays $P^{\text {MIPS }}$ and $P^{\text {all }}$ for three MIPS categories that exhibit large differences between the two PDFs.

Permutations tests were performed to attach statistical significance to the possible rejection of the null hypothesis. For a particular MIPS category represented by $K$ genes the RMS score (from equation 1 ) is compared with the RMS scores obtained by randomly drawing $K$ genes from the distribution of all genes, estimating the PDF based on these points and recomputing equation 1. A permutation score is denoted by $\mathrm{RMS}^{*}$. A total of 50000 permutations (random-drawing experiments) were performed for each MIPS category, resulting in $50000 \mathrm{RMS}^{\star}$ values. The $P$-value for statistical significance is defined as:

$P=\frac{1}{50000} \sum_{i=1}^{50000} I\left(\mathrm{RMS}^{*}(i) \geq \mathrm{RMS}\right)$

where $I($.$) is the indicator function and \operatorname{RMS}^{*}(i)$ the RMS score of the permutated data for the $i$-th permutation. The cut-off to decide whether the null hypothesis should be rejected is set at the 0.05 level. The Benjamini-Hochberg correction for multiple testing (Benjamini \& Hochberg, 1995) was applied, since many MIPS categories were tested. As a consequence of this correction MIPS functional categories are significant when $P<6 \times 10^{-3}$. The more strict Bonferroni correction, which assumes independent tests, was not applied, since there is a large overlap amongst MIPS functional categories. Moreover, only the MIPS functional categories containing five or more genes were tested.

\section{RESULTS}

\section{Acquisition and classification of the protein dataset}

The yeast proteome response to anaerobic growth under steady-state conditions was investigated using metabolic stable-isotope labelling $\left({ }^{14} \mathrm{~N} /{ }^{15} \mathrm{~N}\right)$ to allow relative quantification (Heck \& Krijgsveld, 2004). Proteins were separated by SDS-PAGE, followed by triplicate LC-MS/ MS analyses. Although high-resolution nanoflow liquid chromatography was used, many peptides still co-eluted, which is a well-known phenomenon in such analyses (Liu et al., 2004; Taoka et al., 2004). Therefore a triplicate analysis of the same sample was performed to increase the number of unique peptides being analysed, which is beneficial to the accuracy of both protein identification and quantification. This triplicate analysis resulted in a $48 \%$ increase in the amount of identified proteins compared to a single analysis. In addition, a biologicalreplicate experiment was performed in which the ${ }^{14} \mathrm{~N}$ and ${ }^{15} \mathrm{~N}$ labels were exchanged between aerobic and anaerobic cultures, which were also analysed in triplicate LC-MS/MS experiments. Both biological triplicates resulted in a comprehensive dataset of 1499 identified proteins (Fig. 1), with an average of 28 identified peptides per protein. The 


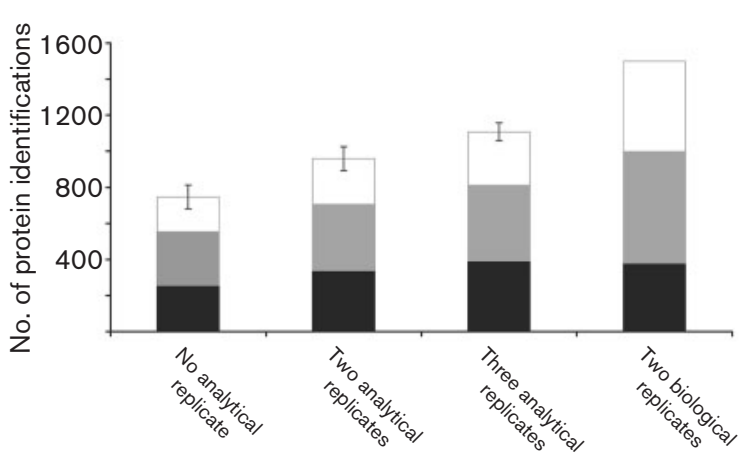

Fig. 1. Replication of analytical and biological experiments increases the number of protein identifications significantly. The bars are divided into three parts; white represents proteins identified solely in aerobic cultures, black represents proteins identified solely in anaerobic cultures, and grey represents proteins identified under both aerobic and anaerobic conditions. Error bars represent the standard deviation of mean values.

false positive rate is $3.03 \%$, with an average of 1.2 identified peptides per protein.

A hypergeometric test (Barash et al., 2001) was used to establish which types of proteins were preferentially detected by this analytical method, and thus to determine the representation of proteins in our dataset compared to the total yeast proteome (MIPS database; http:// mips.gsf.de/). The protein expression groups examined were 'cellular localization' and 'functional categories'. This analysis revealed that the detected proteins are localized for the most part in the cytoplasm, cytoskeleton, nucleus and mitochondria $(P<0.05$; see Supplementary Fig. S1A, available with the online version of this paper). Furthermore, the detected proteins could be assigned to 17 functional categories (see Supplementary Fig. S1B, available with the online version of this paper). The relative over-representation of the detected proteins in these categories may be explained by their high expression levels, as has indeed been previously described for ribosomal proteins, and proteins involved in central carbon metabolism and energy generation (Huh et al., 2003; Kolkman et al., 2006; Prokisch et al., 2004).

\section{Acquiring a robust dataset for relative protein quantification}

Rather stringent settings were used with the RelEx quantification software, and 892 of the 1499 identified proteins showed good-quality extracted chromatograms and peptide spectra (see Supplementary Table S1, available with the online version of this paper). This dataset was further restricted to the proteins that were detected in both biological replicates, which resulted in a dataset of 490 proteins. It can be concluded from Fig. 2(a) that the protein expression ratios originating from the two biological replicates are very similar, although some diverging data points can be observed. These 'outliers' were mainly found for protein ratios based on a small number of peptides; thus the reliability of the dataset could be improved by increasing peptide stringency. However, by doing this, an excessive number of quantified proteins would be discarded, e.g. nearly $75 \%$ of the quantified proteins would be discarded if stringency was set to seven or more quantified peptides per protein in each replicate (Fig. 2b). As an alternative approach, we used multivariate analysis, which revealed that the standard deviation of principal component 2 (PC2) converged towards 0.34 with increasing peptide stringency (Fig. 2c). This value of 0.34 was considered to be the inter-experimental variation of the data points. Thus instead of using a peptide stringency cut-off, this standard deviation of 0.34 was used to set the $95 \%$ confidence interval of PC2 on the initial dataset of 490 quantified proteins. This gave a robust dataset of 418 reliably quantified proteins, which are indicated by triangles in Fig. 2(a). Moreover, the original dataset contained peptides from 56 proteins that were solely detected in samples from one culture condition, of which 23 were unique to the anaerobic and 33 to the aerobic cultures. These could not be processed automatically by the RelEx algorithm, as the program automatically filtered out these data due to poor correlation for the weakly detected peptide of the peptide pair, and therefore had to be manually validated. The final robust dataset consisted of 474 quantified proteins (Supplementary Table S1). We chose to take an arbitrary protein ratio cut-off of twofold, resulting in 137 proteins with a ratio of anaerobic:aerobic $\left(\mathrm{R}_{\mathrm{Anae}}\right.$ : Ae $)$ greater than two considered to be upregulated in the absence of oxygen, while 112 proteins with a $\mathrm{R}_{\mathrm{Anae}} \mathrm{Ae}$ smaller than 0.5 were considered to be downregulated under anaerobiosis (Supplementary Table S1). Many of these 249 changes in protein abundance were expected from current knowledge, although other responses of $S$. cerevisiae to oxygen availability were, to our knowledge, not previously described. The most remarkable proteins, as considered by us, will be discussed.

\section{Protein central carbon metabolism response to yeast anaerobiosis}

The 137 proteins that were expressed at a higher level in the anaerobic cultures were predominantly involved in metabolic functions (92 proteins), while proteins involved in energy generation showed both higher and lower levels (36 and 33 proteins, respectively; Table 1). Some of the observed changes could be anticipated from data on yeast physiology obtained previously, such as the upregulation of the glycolysis and the fermentative pathway. Indeed, in the absence of oxygen, where respiration can no longer occur, substrate-level phosphorylation in glycolysis is essential to sustain cellular growth. To compensate for the low ATP yield [2 versus $16 \mathrm{~mol}$ per mol glucose during respiratory growth (Berg et al., 2007)], the glycolytic flux is tremendously accelerated in anaerobic cultures, as indicated by the five- to sixfold increase in the specific 
Table 1. Key functional categories of up- or downregulated proteins under anaerobiosis

\begin{tabular}{|c|c|c|c|c|}
\hline MIPS category* & Protein identity & $\begin{array}{l}\text { No. of proteins } \\
\text { in cluster } \dagger\end{array}$ & $\begin{array}{l}\text { No. of proteins } \\
\text { in genome } \ddagger\end{array}$ & $P$-value $\S$ \\
\hline \multicolumn{5}{|l|}{ Upregulated proteins } \\
\hline METABOLISM (01) & & 92 & 1520 & $1.3 \times 10^{-10}$ \\
\hline $\begin{array}{l}\text { Amino acid metabolism } \\
(01.01)\end{array}$ & $\begin{array}{l}\text { Gdh3p, His7p, Aro4p, Leu2p, His4p, Thr4p, Hom2p, Lys4p, Sam2p, } \\
\text { Sah1p, Ser3p, Trp2p, Met6p, Met10p, Leu1p, Trp5p, Aro8p, Asn2p, } \\
\text { Ade3p, Arg4p, Ser33p, Ilv3p, Cpa2p, Hom6p, Maelp, Shm2p, } \\
\text { Met17p, Aco1p, Ilv5p, Ilv2p, Arg1p, Ser1p, Gln1p, Tkl1p, Asn1p }\end{array}$ & 35 & 245 & $7.8 \times 10^{-5}$ \\
\hline $\begin{array}{l}\text { Purine nucleotide } \\
\text { anabolism }(01.03 .01 .03)\end{array}$ & $\begin{array}{l}\text { His7p, His4p, Ade5,7p, Ade6p, Ade3p, Imd2p, Ade13p, Ade17p, } \\
\text { Ade4p, Ade2p, Ser1p }\end{array}$ & 11 & 29 & $3.6 \times 10^{-5}$ \\
\hline $\begin{array}{l}\text { C-compound and } \\
\text { carbohydrate utilization } \\
(01.05 .01)\end{array}$ & $\begin{array}{l}\text { Pyk1p, Tps1p, Adh5p, Pgilp, Aro4p, Sam2p, Emi2p, YEL047Cp, } \\
\text { Dld3p, Sah1p, Hxk1p, Pyclp, Hxk2p, Ade3p, Pfk1p, Enolp, } \\
\text { YGR287Cp, Mal12p, Eno2p, Rhr2p, Suc2p, Tdh1p, Tdh2p, Mae1p, } \\
\text { Pgm1p, Gpm1p, Pdc1p, Shm2p, Pdc5p, Acs2p, Acolp, Dak1p, } \\
\text { Pgm2p, Ilv2p, Ade17p, Pfk2p, Gpd2p, Adh1p, Fum1p, Gln1p, } \\
\text { Tkl1p }\end{array}$ & 41 & 388 & $7.9 \times 10^{-5}$ \\
\hline $\begin{array}{l}\text { Other subcategories in } \\
\text { METABOLISM }\end{array}$ & $\begin{array}{l}\text { Imd1p, Pho88p, Cdc48p, Ssb1p, Hem13p, Rib3p, Erg1p, Rnr4p, } \\
\text { Erg11p, Ths1p, Kar2p, Ssclp, Grr1p, Stm1p, Yta12p, Erg2p, Faa4p, } \\
\text { Ssb2p, Cmk2p, Hsp82p }\end{array}$ & 20 & nsll & nsll \\
\hline ENERGY (02) & $\begin{array}{l}\text { Pyk1p, Gdh3p, Tps1p, Adh5p, Pgilp, Dld3p, Hxk1p, Pyc1p, Hxk2p, } \\
\text { Ade3p, Pfk1p, Eno1p, YGR287Cp, Mal12p, Eno2p, Oye2p, Tdh1p, } \\
\text { Aco2p, Tdh2p, Pgm1p, Gpm1p, Pdc5p, Acs2p, Aco1p, Pgm2p, } \\
\text { Asc1p, Pfk2p, Adh1p, Hsp82p, Fum1p, Tkl1p, Rib3p, YEL047Cp, } \\
\text { Yta12p }\end{array}$ & 36 & 369 & $2.4 \times 10^{-2}$ \\
\hline $\begin{array}{l}\text { AMINOACYL-tRNA } \\
\text { SYNTHETASES }(12.10)\end{array}$ & $\begin{array}{l}\text { Ils1p, Grs1p, Ses1p, YDR341Cp, Frs2p, Gus1p, Vas1p, Ded81p, } \\
\text { YHR020Wp, Ths1p, Dps1p, Ala1p }\end{array}$ & 12 & 39 & $2.2 \times 10^{-7}$ \\
\hline \multicolumn{5}{|l|}{ Downregulated proteins } \\
\hline ENERGY (02) & & 33 & 369 & $1.7 \times 10^{-2}$ \\
\hline Electron transport $(02.11)$ & $\begin{array}{l}\text { Cox2p, Atp5p, Qcr7p, Rip1p, Qcr6p, Cox4p, Cox13p, Cox6p, } \\
\text { Cyc1p, Atp7p, Sdh2p, Cox12p, Cyb2p, Cox5Ap, Cyt1p, Atp4p, } \\
\text { Atp20p, Qcr2p }\end{array}$ & 18 & 61 & $6.3 \times 10^{-8}$ \\
\hline Respiration (02.13) & $\begin{array}{l}\text { Atp5p, Gut2p, Cyc1p, Atp7p, Cyb2p, Ald4p, Atp4p, Atp20p, Cox2p, } \\
\text { Pet9p, Qcr7p, Rip1p, Qcr6p, Cox4p, Cox13p, Cox6p, Sdh2p, } \\
\text { Cox12p, Cox5Ap, Cyt1p, Qcr2p }\end{array}$ & 21 & 138 & $1.0 \times 10^{-6}$ \\
\hline $\begin{array}{l}\text { Aerobic respiration } \\
(02.13 .03)\end{array}$ & $\begin{array}{l}\text { Cox2p, Pet9p, Qcr7p, Rip1p, Qcr6p, Cox4p, Cox13p, Cox6p, Sdh2p, } \\
\text { Cox12p, Cox5Ap, Cyt1p, Qcr2p }\end{array}$ & 13 & 77 & $8.3 \times 10^{-5}$ \\
\hline $\begin{array}{l}\text { Other subcategories in } \\
\text { ENERGY }\end{array}$ & $\begin{array}{l}\text { Acs1p, Mdh3p, Kgd2p, Agx1p, Poxlp, Idp2p, Adh2p, Gre2p, Lsclp, } \\
\text { Fdh2p, YPL276Wp, Icl2p }\end{array}$ & 12 & nsll & nsll \\
\hline
\end{tabular}

*Based on the MIPS database (http://mips.gsf.de/genre/proj/yeast).

$\dagger$ Number of up- or downregulated proteins found in the functional category database.

$\ddagger$ Total number of proteins in a particular MIPS category.

$\S P$-values for the over-representation of these groups were calculated using a hypergeometric distribution test.

IIns, remaining members of the specified significant primary MIPS category (energy or metabolism), which were not significantly over-represented in MIPS subcategories.

glucose consumption rate for the anaerobically grown yeast at the same specific growth rate of $0.10 \mathrm{~h}^{-1}$ (DaranLapujade et al., 2004). Glycolytic enzyme activities have previously been shown to be higher in anaerobic cultures of $S$. cerevisiae than in aerobic cultures (Daran-Lapujade et al., 2004; van Hoek et al., 2000). Consistently, 12 of the 17 quantified glycolytic proteins were significantly more abundant under anaerobiosis (Table 1 and Fig. 3a). Downstream glycolysis, the fermentative pathway leading to the conversion of pyruvate to ethanol through two reactions, is catalysed by eight main proteins, of which four were upregulated under anaerobiosis (Fig. 3a). On the other hand, Adh $2 p$ is the favoured enzyme for ethanol utilization rather than ethanol formation, so its reduced expression under anaerobiosis is in agreement with previous studies (Salusjarvi et al., 2003; Tai et al., 2005)

By enzymic assays and ${ }^{13} \mathrm{C}$-labelling studies, the tricarboxylic acid (TCA) cycle has been shown not to function as 


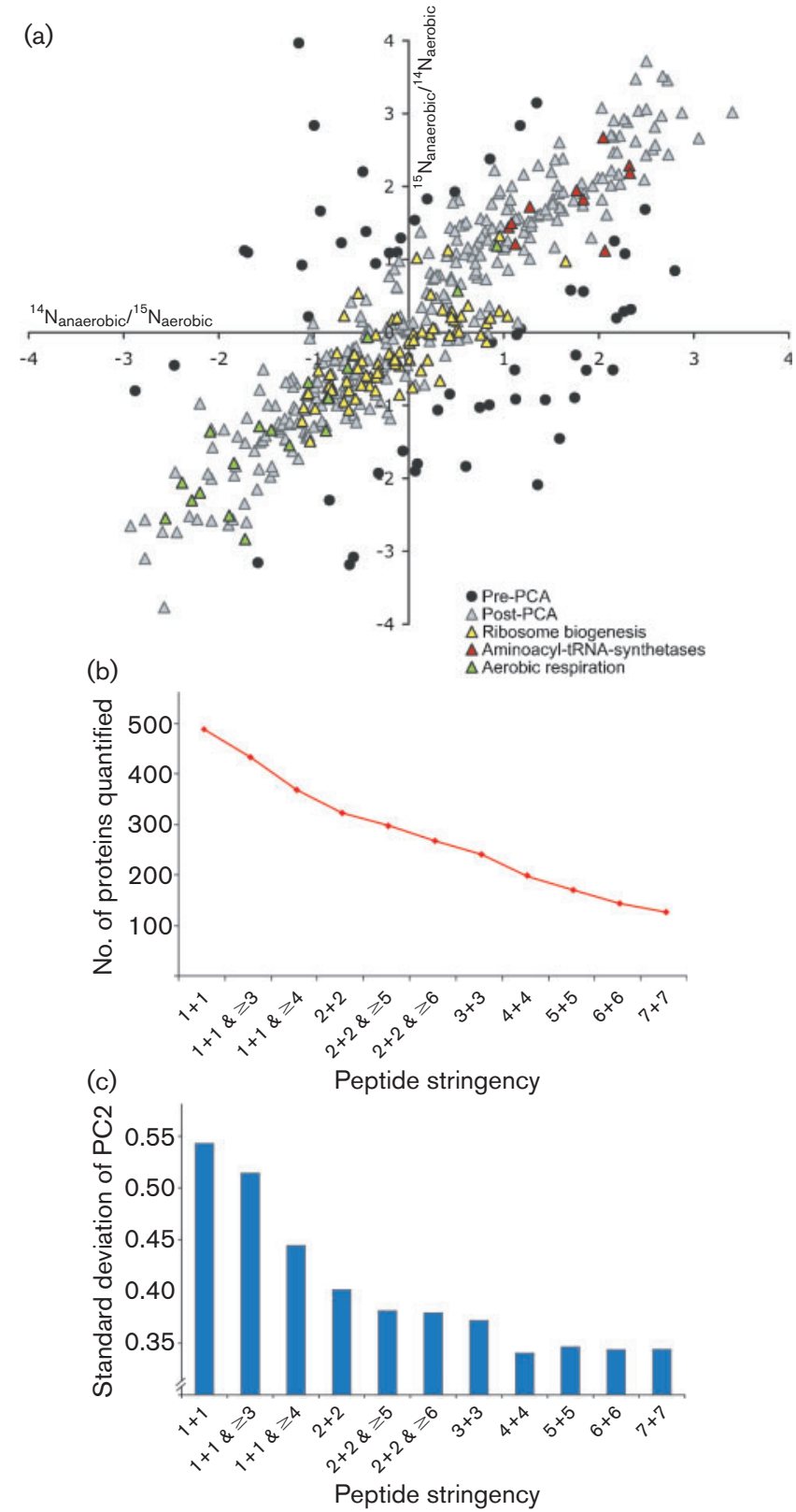

a cycle under oxygen deprivation (Camarasa et al., 2003). However, even in the absence of oxygen, essential precursors such as oxaloacetate and 2-oxoglutarate (e.g. for the synthesis of amino acids) have to be supplied. The TCA cycle is then restricted to an oxidative branch leading to 2-oxoglutarate and a reductive branch leading to oxaloacetate (Camarasa et al., 2003). This scenario is supported by the anaerobic downregulation of Lsc2p, Sdh2p and Kgd2p (Fig. 3a), proteins that belong to the three complexes that convert 2-oxoglutarate to fumarate. Furthermore, increased or unchanged expression of Citlp, Acolp, Aco2p, Idh1p, Idh2p, Pyclp, Fum1p and Frds1p ensured the supply of 2-oxoglutarate via the oxidative branch of the TCA pathway and the production of
Fig. 2. Statistical analysis of the proteomics dataset. (a) Scatter plot of the ratios (anaerobic:aerobic; represented as $\log _{2}-$ transformed ratios) for both experiments: ${ }^{14} \mathrm{~N}_{\text {anaerobic }}$ and ${ }^{15} \mathrm{~N}_{\text {aerobic }}$ and for the reversed experiment ${ }^{15} \mathrm{~N}_{\text {anaerobic }}$ and ${ }^{14} \mathrm{~N}_{\text {aerobic. }}$. The black dots are the values removed after applying PCA; the triangles are the data points that remained. The coloured triangles within the remaining dataset represent quantified proteins from three typical MIPS functional categories: ribosome biogenesis (yellow), aminoacyl-tRNA sythetases (red) and aerobic respiration (green). (b) The number of protein quantifications decreases dramatically with increasing peptide stringency. Peptide stringency reads as follows; e.g. $2+2$, at least two quantified peptides from each biological replicate; $1+1 \&>2$, more than two quantified peptides in total and at least one peptide from either biological replicate, etc. (c) PCA analysis on the total set of 490 quantified proteins, showing the progression of the standard deviation of principal component 2 with increasing peptide stringency. The standard deviation levels off at approximately 0.34 , which may be the value of the inter-experimental variation.

oxaloacetate, malate, fumarate and succinate via the reductive branch (Fig. 3a; Supplementary Table S1).

\section{Responses of proteins involved in respiratory chain, oxidative stress, and processes requiring molecular oxygen}

As expected in the absence of oxygen, many of the respiratory chain complex subunits showed reduced expression levels. These reduced protein levels were found for cytochromes $c_{1}$ (Cytlp) and $b_{2}$ (Cyb2p) and several subunits of the $\mathrm{F}_{1} \mathrm{~F}_{0}$-ATPase (Atp4p, Atp5p, Atp7p and Atp20p), the ubiquinol-cytochrome $c$ reductase (Qcr2p, Qcr6p and Qcr7p) and the cytochrome $c$ oxidases (Cox2p, Cox4p, Cox5Ap, Cox6p, Cox12p and Cox13p). Oxidative stress is typically low under anaerobiosis, which explains why the proteins involved in the oxidative stress response displayed strongly reduced expression. These quantified proteins are the catalase Ctalp, both superoxide dismutases (Sod1p and Sod2p), the glutathione peroxidase Hyrlp, which is involved in oxidative stress signalling, and the protein-methionine-S-oxide reductase Mxrlp. It is noteworthy that Yhblp, suspected to be involved in oxidative stress and previously described as transcriptionally induced by oxygen (Buisson \& Labbe-Bois, 1998; Crawford et al., 1995), displayed no change in expression. Finally, Faa2p and Poxlp, involved in oxygen-dependent $\beta$-oxidation of fatty acids, were also expressed at lower levels in the absence of oxygen (Trotter, 2001). It is remarkable that several haemoproteins were detected not only in aerobic, but also in anaerobic chemostat cultivations (Cytlp, Ccp1p, Erg11p, Ecm17p, Yhb1p and Met10p). The synthesis of haem, a structural component of these proteins, involves two reactions requiring molecular oxygen, Hem13p and Hem14p (Amillet et al., 1995; Camadro \& Labbe, 1996; Glerum et al., 1996) and is therefore assumed not to be functional under anaerobiosis 


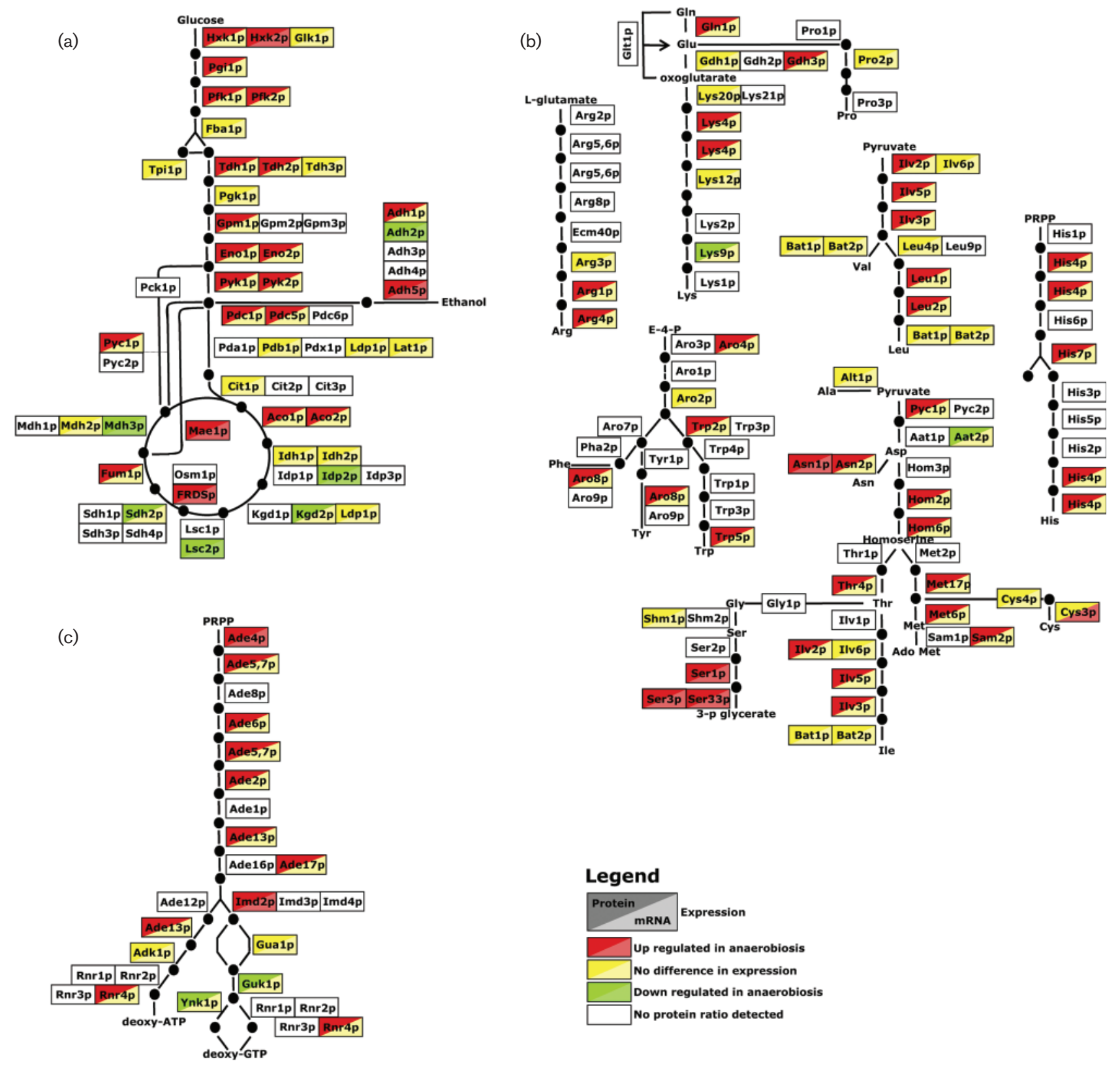

Fig. 3. Differential expression of identified proteins involved in metabolic functions. (a) Glycolysis and TCA cycle. (b) Amino acid biosynthetic pathways. (c) Purine nucleotide anabolism. The upper/left half of the boxes represents the protein expression ratio, the lower/right half represents the mRNA expression ratio. Protein/mRNA levels increased under anaerobiosis are indicated in red, proteins showing no difference in expression are indicated in yellow, protein/mRNA levels decreased under anaerobiosis are shown in green. When a box has no colour the protein was either not detected or did not satisfy our quantification restrictions.

(Porra \& Falk, 1964; Sano \& Granick, 1961). The few reports that describe haem content and the presence of active haemoproteins in anaerobically grown yeast are contradictory (Rosenfeld \& Beauvoit, 2003). It is, however, conceivable that the proteins detected in the present study are inactive apoproteins, synthesized despite the absence of haem, but ready to be matured when oxygen becomes available (Ruis, 1979).

\section{Differential expression of proteins involved in amino acid, purine nucleotide and protein metabolism}

Surprisingly, many enzymes involved in amino acid and purine nucleotide metabolism were expressed at higher levels in the anaerobic cultures (Table 1, Fig. 3b, c). Among the 43 quantified proteins involved in amino acid 

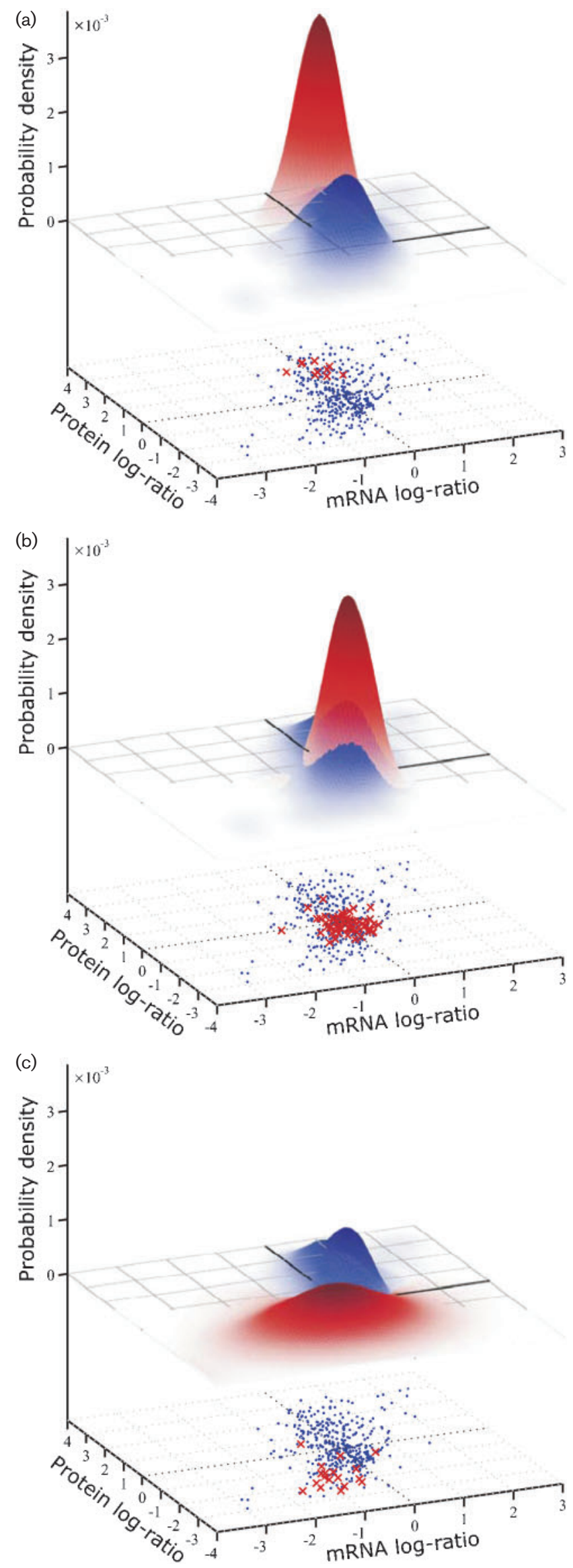

Fig. 4. Three illustrative examples of probability density functions of particularly regulated groups (in red) versus the total dataset (in blue). The data points in the two-dimensional plane below the density functions represent the mRNA and protein ratios of the 404 quantified proteins. The data points belonging to the regulated group (a particular MIPS functional category) are indicated by red crosses; the other points are indicated by blue dots. The density functions have been estimated on the basis of these data points. See Methods for details. The blue density is based on all 404 genes; the red density is based on the genes of a particular functional category: (a) aminoacyl-tRNA synthetases, (b) ribosome biogenesis, and (c) electron transport and membrane-associated energy conservation.

metabolism, having a catalytic activity and a well-defined function, 27 displayed a 2 - to 10 -fold higher abundance under anaerobiosis, 14 remained unchanged, while Lys9p and Aat $2 \mathrm{p}$ were expressed at lower levels (Fig. 3b; Supplementary Table S1). Similarly, out of the 12 quantified enzymes involved in purine synthesis, eight were more abundant in the absence of oxygen (Fig. 3c and Supplementary Table S1). Only one protein (Ynk1p), which converts GDP into GTP, displayed a lower abundance. Considerably higher protein levels under anaerobiosis were unexpectedly detected for 11 , mostly cytosolic, aminoacyl-tRNA synthetases. The regulation of aminoacyl-tRNA synthetase expression in yeast is still largely unknown and, to our knowledge, this is the first report of regulation by oxygen availability. In order to see if oxygen depletion led to a more general effect on translation, we screened the other quantified proteins involved in this cellular process. Among the 62 quantified ribosomal proteins, the expression of the large majority (58 proteins) was not affected by oxygen availability. In contrast, the level of all three quantified subunits of the pentameric ribosomal stalk complex (Rpp1Ap, Rpp1Bp and Rpp2Bp) was lower in the absence of oxygen. Conversely, the expression of several proteins involved in translation initiation (Niplp, Ded1p, Pablp, Rpglp and eIF3 subunits), in translation elongation (Teflp, Tef4p, Eftlp and Yef3p), in chaperoning the folding of nascent chains (i.e. the tripartite complex consisting of Ssblp, Ssb2p and Ssz1p) (Gautschi et al., 2002), and in translation efficiency (Asclp) (Chantrel et al., 1998) was higher under anaerobic conditions. Regarding protein degradation, Pre9p, Rpt1p, Rpn9p, Rpn12p and Rpn13p, which are five regulatory subunits of the proteasome known to be upregulated under oxidative stress (Haugen et al., 2004), were also in our study detected at a higher level under anaerobic conditions, where oxidative stress is typically low.

\section{Protein levels versus transcript level}

Several specific pathways were reprogrammed under anaerobiosis. In order to investigate the regulatory events involved in this reprogramming, we compared the protein data with available transcript data from Tai et al. (2005). 
We applied identical culture conditions for both the transcriptome and proteome analyses, which is very important to minimize experimental discrepancies between the two datasets. We employed an approach using functional categories of proteins, instead of investigating the transcriptional and post-transcriptional mechanisms involved in the regulation of each individual protein. We assessed whether proteins belonging to specific yeast functional categories were enriched in a specific part of the data space covering the protein ratios versus the mRNA ratios. More specifically, both the statistical significance of the differences between the distribution of all points in the $\mathrm{mRNA}$ /protein dataset, and the distribution of points corresponding to a functional category, were considered. This resulted in the identification of 17 functional categories with sizes ranging from 10 to 173 proteins, for which most of the members showed a consistent pattern for the regulation at the transcript and/or protein level in response to the presence of oxygen (see Supplementary Table S2, available with the online version of this paper). The density plots of three illustrative categories are represented in Fig. 4, showing that changes in expression of the transcripts and proteins belonging to the functional categories aminoacyl-tRNA synthetases, ribosome biogenesis and electron transport and membrane-associated energy conservation (shown in red) are clearly clustered within a specific and distinct section of the density plot compared to the total dataset (shown in blue). Such a test, although enabling the efficient identification of regulatory trends for the functional categories discussed, does not provide direct details about the type of regulation involved, i.e. either transcriptional or post-transcriptional regulation. The changes in mRNA and protein expression of these categories with consistent expression profiles were therefore represented in box plots, to gain improved insights into the level at which regulation occurs and to test their statistical relevance (Fig. 5). The distributions of the protein and the mRNA ratios are shown in light and dark grey, respectively. Boxes whose notches do not overlap indicate that the medians of the protein and the mRNA ratios are different at the $5 \%$ significance level, as for the first MIPS category shown in Fig. 5, purine nucleotide anabolism. Fig. 5 illustrates clearly that the ribosome biogenesis and RNA binding categories were not affected, at either the mRNA or the protein level, despite the major physiological changes triggered by the availability of oxygen. Ribosome biogenesis involves a rather large group of proteins (63 quantified proteins) that displays a tight distribution, indicating homogeneity in the regulation of the ribosomal transcripts and proteins. Although the ratios are much more widely distributed for four other functional categories, i.e. purine nucleotide metabolism, energy, electron and hydrogen transport and respiration, the notches of the boxes overlap, so no distinction between transcriptional or post-transcriptional regulation can be made. In contrast, the large category of metabolism (173 quantified proteins) is characterized by a small but significant difference in the regulation of transcript- and

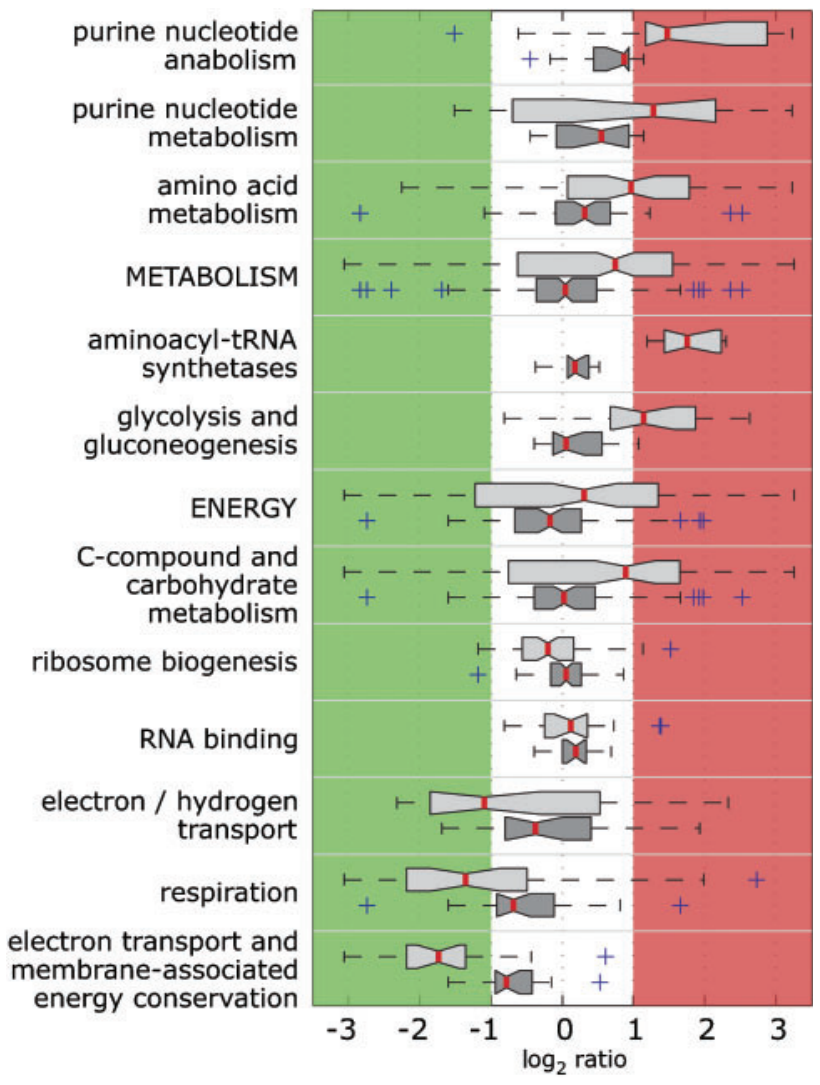

Fig. 5. Boxplot of mRNA and protein ratios for the significant functional categories as determined from the density test. The protein and mRNA ratios are represented by the light grey and dark grey boxes, respectively. The boundaries of a box indicate the lower and upper quartile values; the line represents the median. The whiskers (the lines extending from each end of the boxes) show the extent of the rest of the data. Outliers are data with values beyond the ends of the whiskers, indicated by ' + '. The notches of the box represent a robust estimate of the uncertainty about the medians for box-to-box comparison. Boxes whose notches do not overlap indicate that the medians of the two groups are different at the $5 \%$ significance level. Data points in the green and red areas correspond to anaerobically decreased and increased levels, respectively. Four categories (ribosomal proteins, C-compound and carbohydrate utilization, C-compound carbohydrate anabolism, and aerobic respiration) overlap markedly with some of the other 13 identified categories, and are therefore not represented.

protein expression. The distribution of this large group mostly results from three of its subcategories: purine nucleotide anabolism, amino acid metabolism and glycolysis/gluconeogenesis. As can be seen from Fig. 5, the transcript levels of the 94 quantified members of these three categories remained stable under anaerobiosis, while the levels of their protein counterparts significantly changed, suggesting that expression was strongly regulated at the protein level. Finally, the data in Fig. 5 also strongly indicate that the proteins involved in aminoacyl-tRNA synthesis and electron transport/membrane-associated 
energy conservation are regulated by post-transcriptional events. This box plot analysis therefore suggests that many proteins belonging to metabolic pathways or having similar functions were specifically and concertedly regulated at the post-transcriptional level, which may be at all levels between protein synthesis and degradation.

\section{DISCUSSION}

\section{Chemostats allow proper systems biology}

Excellent reproducibility, and the option to manipulate individual culture parameters, make chemostat cultivation very well suited to quantitative systems biology research (Bro et al., 2003; Daran-Lapujade et al., 2004; Jansen et al., 2005; Kolkman et al., 2006). In particular, chemostat cultivation enables researchers to dissect effects of specific growth rate and of other parameters. This is not possible in batch cultures, where changes in growth conditions can have a drastic impact on the specific growth rate. Moreover, chemostats eliminate the intrinsic dynamics of conventional batch cultures, which further facilitates an uncluttered comparison of different cellular information levels. In the present study, we exploited these advantages in a chemostat-based proteome and transcriptome analysis of the response of S. cerevisiae to oxygen availability. Based on metabolic stable-isotope labelling and LC/MS-MS analysis, approximately 1500 proteins were identified. A thorough statistical analysis of biological and analytical replicates enabled the reliable relative quantification of 474 proteins in aerobic and anaerobic cultures. This provides a valuable advance compared to previous studies on yeast responses to oxygen availability that rested solely on transcriptome analysis (Kwast et al., 1998; Tai et al., 2005; ter Linde et al., 1999) or focused on small subsets of proteins (Rosenfeld \& Beauvoit, 2003; Salusjarvi et al., 2003). New and intriguing differences have been uncovered, while the differential expression of other proteins in response to oxygen availability was anticipated from previously published results. Several of these differences involved central metabolic processes and will be discussed below.

\section{Post-transcriptional regulation of glycolysis}

The in vivo activity of glycolysis, the central pathway of sugar metabolism in yeast, is much higher in anaerobic cultures than in aerobic cultures (van Hoek et al., 2000; Verduyn et al., 1990). While the levels of most of the 21 quantified glycolytic proteins were 2- to 10 -fold higher in the anaerobic cultures than in the aerobic cultures, the corresponding mRNA levels remained essentially the same (Supplementary Table S1). These observations confirm and extend a previous study, which showed that, under a number of cultivation conditions, glycolytic mRNAs in yeast are a poor indicator of glycolytic flux (DaranLapujade et al., 2004). Instead, the present study suggests that modulations at the post-transcriptional level play a major role in the regulation of yeast glycolysis. Whereas transcriptional regulation of glycolytic genes has been extensively studied, very little is known about posttranscriptional regulation of glycolytic and fermentative protein levels, which can be, for example, at the translation or degradation step. Sophisticated mechanisms involved in regulation of levels of a few yeast proteins have been demonstrated previously, most notably for Gcn4p (Hinnebusch, 2005). Our study stresses the necessity to investigate the molecular mechanisms involved in the regulation of translation and/or degradation of glycolytic proteins.

Glycolytic enzymes are among the most abundant proteins in yeast. In aerobic, glucose-limited chemostat cultures, they have been estimated to represent $\sim 8 \%$ of the total cellular protein in S. cerevisiae (van Hoek, 2000) and, based on the present data, this contribution increases to $21 \%$ in anaerobic cultures. This implies that a small subset of proteins uses a sizeable fraction of the overall translation rate, which may require adaptations to the protein synthetic machinery. Indeed, several changes in key proteins were observed that may affect protein biosynthesis.

\section{Aminoacyl-tRNA synthetases}

Levels of all quantified aminoacyl-tRNA synthetases (aaRS) were significantly higher under anaerobiosis (Table 1). As observed for the glycolytic proteins, the increased levels of aaRS in the anaerobic cultures were not due to increased levels of their corresponding mRNA. Because of their central role in translation, the synthesis rate of aaRS is assumed to be tightly controlled (Romby et al., 1996), for instance to prevent the risk of misacylation of tRNAs as a result of excessive aaRS expression, as has been shown previously for AspRS (Ryckelynck et al., 2003). Regulatory mechanisms for aaRS have been described previously for bacteria (Putzer et al., 1995; Romby et al., 1996) and have recently been identified in yeast. The previous observation that multi-copy overexpression of AspRS from the strong constitutive GPD promoter did not lead to increased AspRS production suggested that AspRS expression was regulated post-transcriptionally (Frugier et al., 2005). AspRS has been shown to interact with the $5^{\prime}$ end of its own mRNA, which resulted in a decrease in the level of a fused reporter protein. A model for translational regulation of AspRS assumes the nuclear import of excess AspRS and its competitive binding to newly transcribed tRNA ${ }^{\text {Asp }}$ and $\mathrm{mRNA}^{\text {Asp }}$, thus reducing its own expression (Frugier et al., 2005). Although reduced expression of AspRS could also be the consequence of accelerated degradation of AspRSbound mRNA $^{\text {Asp }}$, experimental evidence does not support this hypothesis (Frugier et al., 2005). We observed a 3.2-fold increase in the protein level of AspRS under anaerobiosis, while its transcript level remained unchanged. If the regulation of mRNA turnover was involved in the regulation of AspRS expression, a difference in its transcript level 
should have been observed. While most previous studies have specifically focused on AspRS, our results indicate that regulation at the protein level is likely to be a general phenomenon of aaRS in S. cerevisiae, which involves at least the 12 relatively quantified aaRS proteins in our dataset (Table 1).

A previous comparison of the proteomes of carbon- and nitrogen-limited yeast cultures, in which the intracellular amino acid availability may be expected to be different, did not reveal a change in expression of aaRS (Kolkman et al., 2006). A similar constant expression of aaRS was found in yeast cells grown on rich and minimal media (Washburn et al., 2003b). Conversely, in cultures growing on the fermentable carbon source galactose, the abundance of three aaRS (out of five quantified) was twofold higher than in slowly growing cultures on ethanol (Griffin et al., 2002). These studies point at a positive correlation of aaRS expression and glycolytic activity.

\section{Amino acid and purine nucleotide synthesis}

The strongly increased levels of proteins involved in amino acid and purine nucleotide biosynthesis were another set of results that were unexpected on the basis of previous transcriptome studies (Lai et al., 2005; Tai et al., 2005; ter Linde et al., 1999) and could be attributed to posttranscriptional regulation. Transcriptional regulation of these two pathways has been extensively investigated and involves a common regulation via Gcn4p (general amino acid control) and Bas1p/Bas2p in response to nutrient availability (Daignan-Fornier \& Fink, 1992; Hinnebusch, 2005). Although post-transcriptional regulation of these pathways has not been investigated systematically, some isolated observations support our data. A previous study that compared proteome and transcriptome levels for the yeast response to different carbon sources showed that for Ade5,7p the protein level had increased, while the corresponding mRNA level remained unchanged (Griffin et al., 2002). Also Gln1p synthesis was previously proposed to be regulated post-transcriptionally, on the basis of both large- and small-scale studies. In addition, most of the nine enzymes involved in amino acid biosynthesis that were found previously to be differentially expressed in response to carbon source were post-transcriptionally regulated (Griffin et al., 2002; Kolkman et al., 2006). These independent studies strengthen the case for involvement of post-transcriptional control of amino acid and purine nucleotide biosynthesis.

\section{Translational machinery}

Among 62 quantified ribosomal proteins (Supplementary Table S1), the protein levels of 58 were not affected by oxygen availability. Expression of ribosomal proteins is known to be growth-rate-dependent (Kief \& Warner, 1981) and the detected stable levels are therefore consistent with the experimental set-up, in which the specific growth rate was fixed at $0.10 \mathrm{~h}^{-1}$. The identical specific growth rates and the small differences between the total protein contents of the aerobic and anaerobic chemostat cultures $(42 \pm 1 \%$ and $45 \pm 1 \%$, respectively) indicated that the total protein synthesis rates under these conditions differed by less than $10 \%$. Moreover, total RNA, which consists mainly of rRNA, has also been purified from these cultures and relatively quantified, which revealed that no change in rRNA content occurred under anaerobiosis (results not shown). This confirms that the core translational machinery is maintained. Surprisingly, the levels of 14 proteins involved in translation initiation and elongation, as well as in processing of nascent proteins, were higher under anaerobic conditions. In addition, proteins of the ribosomal stalk complex, which has been previously suggested to regulate translation rate (Ballesta \& Remacha, 1996), were downregulated. The stalk complex is formed by four small acidic polypeptides and one larger protein and is a universal feature of ribosomes of all organisms (Ballesta et al., 1999). In yeast, as in bacteria, it is most probably involved in interactions between translation factors and ribosomes and in the regulation of translation; however, its precise role is still unclear (Ballesta \& Remacha, 1996; Liljas, 1982).

\section{Potential roles of protein demand and amino acid supply in the regulation of protein synthesis}

The differential expression of approximately 60 proteins related to protein synthesis in response to oxygen availability suggests a common underlying physiological mechanism. Proteins involved in the fuelling of translation (amino acids, purine nucleotides and S-adenosylmethionine) as well as in translation itself (aaRS and translation factors) were detected at higher levels in the anaerobic cultures. While, at first glance, this reprogramming might suggest an enhanced rate of translation, the overall translation rate was in fact equal under aerobic and anaerobic conditions, unless protein degradation rates were drastically different. We indeed detected increased levels of five proteasome regulatory proteins; however, their significance in enhanced protein degradation is not known.

One factor in the observed changes in protein synthesis might be the faster synthesis rate of abundant glycolytic proteins under anaerobic conditions. This would then imply the existence of mechanisms that specifically enhance the translation of subsets of mRNAs that are in high demand under specific cultivation conditions. Such specificity has been shown for proteins like Ded1p, which regulate the translation of specific mRNA species (Noueiry et al., 2000).

Another possible factor is the perturbation of the central carbon metabolism. The different in vivo glycolytic fluxes in the aerobic and anaerobic cultures may result in different intracellular concentrations of low-molecularmass metabolites in central metabolism. Several of these compounds are precursors for amino acid biosynthesis, 
and altered concentrations therefore may well affect intracellular amino acid pools. Upregulation of amino acid synthesis and aaRS would then reflect a cellular response to decreased amino acid availability. In addition to precursor synthesis, oxygen availability may affect the cellular amino acid status as a result of metabolic compartmentation. Several proteins involved in amino acid synthesis are localized in the mitochondria (Huh et al., 2003; Kumar et al., 2002; Ryan et al., 1973). In the absence of oxygen, the function and morphology of mitochondria are strongly affected (Visser et al., 1994, 1995) and may contribute to changes in the cytosolic amino acid pools available for protein synthesis.

\section{Concluding remarks}

The response to oxygen availability was analysed using differential protein analysis on metabolically-stable-isotope-labelled S. cerevisiae. While it could be anticipated that levels of glycolytic and respiratory chain proteins would change, unexpected and intriguing differences were found for proteins involved in other cellular processes. Since it is not known how these processes are regulated, well-controlled chemostats were used that allowed a detailed comparison/analysis of proteome versus transcriptome responses of $S$. cerevisiae to anaerobiosis. This has yielded strong evidence of post-transcriptional regulation of central cellular processes, including glycolysis, aminoacyl tRNA synthesis and amino acid biosynthesis. Our study demonstrates the necessity to integrate information along the pathway from gene to in vivo flux. Such integrated approaches can only be reliable if wellcontrolled and reproducible culture conditions are used and robust datasets are created. While our data do not enable mechanistic explanations, they provide guidance for new biochemical studies into the underlying processes. Future work should include investigation of translation and protein turnover rates and signal transduction pathways, and accurate analysis of intracellular metabolites, including amino acids. In compartmented eukaryotic cells especially, this is still a formidable challenge.

\section{ACKNOWLEDGEMENTS}

We thank Dr A. Kolkman and Ing. J. Barends (Utrecht University) for technical assistance with the analyses on the yeast proteome and Dr D. de Ridder (Delft University of Technology) for helpful comments about data analysis. We also thank Dr J. R. Yates and Dr M. J. MacCoss for their help and instructions using the RelEx software in our laboratory. M.J.L.d.G. acknowledges the Kluyver Centre for Genomics of Industrial Fermentation (http://www.kluyvercentre.nl/) for financial support. This work was also supported by the Netherlands Proteomics Centre (http://www.netherlandsproteomicscentre.nl/).

\section{REFERENCES}

Amillet, J. M., Buisson, N. \& Labbe-Bois, R. (1995). Positive and negative elements involved in the differential regulation by heme and oxygen of the HEM13 gene (coproporphyrinogen oxidase) in Saccharomyces cerevisiae. Curr Genet 28, 503-511.

Ballesta, J. P. \& Remacha, M. (1996). The large ribosomal subunit stalk as a regulatory element of the eukaryotic translational machinery. Prog Nucleic Acid Res Mol Biol 55, 157-193.

Ballesta, J. P., Rodriguez-Gabriel, M. A., Bou, G., Briones, E., Zambrano, R. \& Remacha, M. (1999). Phosphorylation of the yeast ribosomal stalk. Functional effects and enzymes involved in the process. FEMS Microbiol Rev 23, 537-550.

Barash, Y., Bejenaro, G. \& Friedman, N. (2001). A simple hypergeometric approach for discovering putative transcription factor binding sites. In Algorithms in Bioinformatics, First International Workshop, WABI 2001, Aarhus, Denmark, August 28-31, 2001, Proceedings, pp. 278-293. Heidelberg: Springer Berlin.

Benjamini, Y. \& Hochberg, Y. (1995). Controlling the false discovery rate: a practical and powerful approach to multiple testing. $J R$ Stat Soc, Ser B, Methodol 57, 289-300.

Berg, J., Tymoczko, J. \& Stryer, L. (2007). Biochemistry, 6th edn. New York: W.H. Freeman.

Bro, C., Regenberg, B., Lagniel, G., Labarre, J., Montero-Lomeli, M. \& Nielsen, J. (2003). Transcriptional, proteomic, and metabolic responses to lithium in galactose-grown yeast cells. J Biol Chem 278, 32141-32149.

Buisson, N. \& Labbe-Bois, R. (1998). Flavohemoglobin expression and function in Saccharomyces cerevisiae. No relationship with respiration and complex response to oxidative stress. J Biol Chem 273, 9527-9533.

Camadro, J. M. \& Labbe, P. (1996). Cloning and characterization of the yeast HEM14 gene coding for protoporphyrinogen oxidase, the molecular target of diphenyl ether-type herbicides. J Biol Chem 271, 9120-9128.

Camarasa, C., Grivet, J. P. \& Dequin, S. (2003). Investigation by ${ }^{13} \mathrm{C}-$ NMR and tricarboxylic acid (TCA) deletion mutant analysis of pathways for succinate formation in Saccharomyces cerevisiae during anaerobic fermentation. Microbiology 149, 2669-2678.

Chantrel, Y., Gaisne, M., Lions, C. \& Verdiere, J. (1998). The transcriptional regulator Haplp (Cyplp) is essential for anaerobic or heme-deficient growth of Saccharomyces cerevisiae: genetic and molecular characterization of an extragenic suppressor that encodes a WD repeat protein. Genetics 148, 559-569.

Crawford, M. J., Sherman, D. R. \& Goldberg, D. E. (1995). Regulation of Saccharomyces cerevisiae flavohemoglobin gene expression. J Biol Chem 270, 6991-6996.

Daignan-Fornier, B. \& Fink, G. R. (1992). Coregulation of purine and histidine biosynthesis by the transcriptional activators BAS1 and BAS2. Proc Natl Acad Sci U S A 89, 6746-6750.

Daran-Lapujade, P., Jansen, M. L., Daran, J. M., van Gulik, W., de Winde, J. H. \& Pronk, J. T. (2004). Role of transcriptional regulation in controlling fluxes in central carbon metabolism of Saccharomyces cerevisiae. A chemostat culture study. J Biol Chem 279, 9125-9138.

Duin, R. P. W. (1976). On the choice of smoothing parameters for Parzen estimators of probability density functions. IEEE Trans Comput C-25, 1175-1179.

Eng, J. K., McCormack, A. L. \& Yates, J. R., III (1994). An approach to correlate tandem mass spectral data of peptides with amino acid sequences in a protein database. J Am Soc Mass Spectrom 5, 976-989.

Frugier, M., Ryckelynck, M. \& Giege, R. (2005). tRNA-balanced expression of a eukaryal aminoacyl-tRNA synthetase by an mRNAmediated pathway. EMBO Rep 6, 860-865.

Gautschi, M., Mun, A., Ross, S. \& Rospert, S. (2002). A functional chaperone triad on the yeast ribosome. Proc Natl Acad Sci U S A 99, 4209-4214. 
Glerum, D. M., Shtanko, A., Tzagoloff, A., Gorman, N. \& Sinclair, P. R. (1996). Cloning and identification of HEM14, the yeast gene for mitochondrial protoporphyrinogen oxidase. Yeast 12, 1421-1425.

Griffin, T. J., Gygi, S. P., Ideker, T., Rist, B., Eng, J., Hood, L. \& Aebersold, R. (2002). Complementary profiling of gene expression at the transcriptome and proteome levels in Saccharomyces cerevisiae. Mol Cell Proteomics 1, 323-333.

Haugen, A. C., Kelley, R., Collins, J. B., Tucker, C. J., Deng, C., Afshari, C. A., Brown, J. M., Ideker, T. \& Van Houten, B. (2004). Integrating phenotypic and expression profiles to map arsenicresponse networks. Genome Biol 5, R95.

Heck, A. J. \& Krijgsveld, J. (2004). Mass spectrometry-based quantitative proteomics. Expert Rev Proteomics 1, 317-326.

Hinnebusch, A. G. (2005). Translational regulation of GCN4 and the general amino acid control of yeast. Annu Rev Microbiol 59, 407-450.

Huh, W. K., Falvo, J. V., Gerke, L. C., Carroll, A. S., Howson, R. W., Weissman, J. S. \& O'Shea, E. K. (2003). Global analysis of protein localization in budding yeast. Nature 425, 686-691.

Jansen, M. L., Diderich, J. A., Mashego, M., Hassane, A., de Winde, J. H., Daran-Lapujade, P. \& Pronk, J. T. (2005). Prolonged selection in aerobic, glucose-limited chemostat cultures of Saccharomyces cerevisiae causes a partial loss of glycolytic capacity. Microbiology 151, $1657-1669$.

Kief, D. R. \& Warner, J. R. (1981). Coordinate control of syntheses of ribosomal ribonucleic acid and ribosomal proteins during nutritional shift-up in Saccharomyces cerevisiae. Mol Cell Biol 1, 1007-1015.

Kolkman, A., Olsthoorn, M. M., Heeremans, C. E., Heck, A. J. \& Slijper, M. (2005). Comparative proteome analysis of Saccharomyces cerevisiae grown in chemostat cultures limited for glucose or ethanol. Mol Cell Proteomics 4, 1-11.

Kolkman, A., Daran-Lapujade, P., Fullaondo, A., Olsthoorn, M. M., Pronk, J. T., Slijper, M. \& Heck, A. J. (2006). Proteome analysis of yeast response to various nutrient limitations. Mol Syst Biol 2, 2006.0026.

Krijgsveld, J., Gauci, S., Dormeyer, W. \& Heck, A. J. (2006). In-gel isoelectric focusing of peptides as a tool for improved protein identification. J Proteome Res 5, 1721-1730.

Kumar, A., Agarwal, S., Heyman, J. A., Matson, S., Heidtman, M., Piccirillo, S., Umansky, L., Drawid, A., Jansen, R. \& other authors (2002). Subcellular localization of the yeast proteome. Genes Dev 16, 707-719.

Kwast, K. E., Burke, P. V. \& Poyton, R. O. (1998). Oxygen sensing and the transcriptional regulation of oxygen-responsive genes in yeast. J Exp Biol 201, 1177-1195.

Kwast, K. E., Lai, L. C., Menda, N., James, D. T., III, Aref, S. \& Burke, P. V. (2002). Genomic analyses of anaerobically induced genes in Saccharomyces cerevisiae: functional roles of Roxlp and other factors in mediating the anoxic response. J Bacteriol 184, 250-265.

Lai, L. C., Kosorukoff, A. L., Burke, P. V. \& Kwast, K. E. (2005). Dynamical remodeling of the transcriptome during short-term anaerobiosis in Saccharomyces cerevisiae: differential response and role of Msn2p and/or Msn4p and other factors in galactose and glucose media. Mol Cell Biol 25, 4075-4091.

Liljas, A. (1982). Structural studies of ribosomes. Prog Biophys $\mathrm{Mol}$ Biol 40, 161-228.

Liu, H., Sadygov, R. G. \& Yates, J. R., III (2004). A model for random sampling and estimation of relative protein abundance in shotgun proteomics. Anal Chem 76, 4193-4201.

MacCoss, M. J., Wu, C. C., Liu, H., Sadygov, R. \& Yates, J. R., III (2003). A correlation algorithm for the automated quantitative analysis of shotgun proteomics data. Anal Chem 75, 6912-6921.
Machado, A., Nunez de Castro, I. \& Mayor, F. (1975). Isocitrate dehydrogenases and oxoglutarate dehydrogenase activities of baker's yeast grown in a variety of hypoxic conditions. Mol Cell Biochem 6, 93-100.

Meiring, H. D., van der Heeft, E., ten Hove, G. J., \& de Jong, A. P. J. M. (2002). Nanoscale $\mathrm{LC}_{\mathrm{M}} \mathrm{MS}^{(n)}$ : technical design and applications to peptide and protein analysis. J Separation Sci 25, 557-568.

Noueiry, A. O., Chen, J. \& Ahlquist, P. (2000). A mutant allele of essential, general translation initiation factor DED1 selectively inhibits translation of a viral mRNA. Proc Natl Acad Sci U S A 97, 12985-12990.

Piper, M. D., Daran-Lapujade, P., Bro, C., Regenberg, B., Knudsen, S., Nielsen, J. \& Pronk, J. T. (2002). Reproducibility of oligonucleotide microarray transcriptome analyses. An interlaboratory comparison using chemostat cultures of Saccharomyces cerevisiae. J Biol Chem 277, 37001-37008.

Porra, R. J. \& Falk, J. E. (1964). The enzymic conversion of coproporphyrinogen 3 into protoporphyrin 9. Biochem J 90, 69-75.

Postma, E., Verduyn, C., Scheffers, W. A. \& Van Dijken, J. P. (1989). Enzymic analysis of the Crabtree effect in glucose-limited chemostat cultures of Saccharomyces cerevisiae. Appl Environ Microbiol 55, 468-477.

Prokisch, H., Scharfe, C., Camp, D. G., II, Xiao, W., David, L., Andreoli, C., Monroe, M. E., Moore, R. J., Gritsenko, M. A. \& other authors (2004). Integrative analysis of the mitochondrial proteome in yeast. PLoS Biol 2, e160.

Putzer, H., Laalami, S., Brakhage, A. A., Condon, C. \& GrunbergManago, M. (1995). Aminoacyl-tRNA synthetase gene regulation in Bacillus subtilis: induction, repression and growth-rate regulation. Mol Microbiol 16, 709-718.

Romby, P., Caillet, J., Ebel, C., Sacerdot, C., Graffe, M., Eyermann, F., Brunel, C., Moine, H., Ehresmann, C. \& other authors (1996). The expression of Escherichia coli threonyl-tRNA synthetase is regulated at the translational level by symmetrical operator-repressor interactions. EMBO J 15, 5976-5987.

Rosenfeld, E. \& Beauvoit, B. (2003). Role of the non-respiratory pathways in the utilization of molecular oxygen by Saccharomyces cerevisiae. Yeast 20, 1115-1144.

Ruis, H. (1979). The biosynthesis of catalase. Can J Biochem 57, 1122-1130.

Ryan, E. D., Tracy, J. W. \& Kohlhaw, G. B. (1973). Subcellular localization of the leucine biosynthetic enzymes in yeast. J Bacteriol 116, 222-225.

Ryckelynck, M., Giege, R. \& Frugier, M. (2003). Yeast tRNA ${ }^{\text {Asp }}$ charging accuracy is threatened by the $\mathrm{N}$-terminal extension of aspartyl-tRNA synthetase. J Biol Chem 278, 9683-9690.

Salusjarvi, L., Poutanen, M., Pitkanen, J. P., Koivistoinen, H., Aristidou, A., Kalkkinen, N., Ruohonen, L. \& Penttila, M. (2003). Proteome analysis of recombinant xylose-fermenting Saccharomyces cerevisiae. Yeast 20, 295-314.

Sano, S. \& Granick, S. (1961). Mitochondrial coproporphyrinogen oxidase and protoporphyrin formation. J Biol Chem 236, 1173-1180.

Servouse, M. \& Karst, F. (1986). Regulation of early enzymes of ergosterol biosynthesis in Saccharomyces cerevisiae. Biochem J 240, 541-547.

Tai, S. L., Boer, V. M., Daran-Lapujade, P., Walsh, M. C., de Winde, J. H., Daran, J. M. \& Pronk, J. T. (2005). Two-dimensional transcriptome analysis in chemostat cultures. Combinatorial effects of oxygen availability and macronutrient limitation in Saccharomyces cerevisiae. J Biol Chem 280, 437-447.

Taoka, M., Yamauchi, Y., Shinkawa, T., Kaji, H., Motohashi, W., Nakayama, H., Takahashi, N. \& Isobe, T. (2004). Only a small subset 
of the horizontally transferred chromosomal genes in Escherichia coli are translated into proteins. Mol Cell Proteomics 3, 780-787.

ter Linde, J. J., Liang, H., Davis, R. W., Steensma, H. Y., van Dijken, J. P. \& Pronk, J. T. (1999). Genome-wide transcriptional analysis of aerobic and anaerobic chemostat cultures of Saccharomyces cerevisiae. J Bacteriol 181, 7409-7413.

Trotter, P. J. (2001). The genetics of fatty acid metabolism in Saccharomyces cerevisiae. Annu Rev Nutr 21, 97-119.

van Hoek, W. P. M. (2000). Fermentative Capacity in Aerobic Cultures of Bakers' Yeast, Delft: Technical University Delft.

van Hoek, P., van Dijken, J. P. \& Pronk, J. T. (2000). Regulation of fermentative capacity and levels of glycolytic enzymes in chemostat cultures of Saccharomyces cerevisiae. Enzyme Microb Technol 26, 724-736.

van Maris, A. J., Luttik, M. A., Winkler, A. A., van Dijken, J. P. \& Pronk, J. T. (2003). Overproduction of threonine aldolase circumvents the biosynthetic role of pyruvate decarboxylase in glucose-limited chemostat cultures of Saccharomyces cerevisiae. Appl Environ Microbiol 69, 2094-2099.

Verduyn, C., Postma, E., Scheffers, W. A. \& van Dijken, J. P. (1990). Physiology of Saccharomyces cerevisiae in anaerobic glucose-limited chemostat cultures. J Gen Microbiol 136, 395-403.

Verduyn, C., Postma, E., Scheffers, W. A. \& Van Dijken, J. P. (1992). Effect of benzoic acid on metabolic fluxes in yeasts: a continuousculture study on the regulation of respiration and alcoholic fermentation. Yeast 8, 501-517.
Visser, W., Scheffers, W. A., Batenburg-van der Vegte, W. H. \& van Dijken, J. P. (1990). Oxygen requirements of yeasts. Appl Environ Microbiol 56, 3785-3792.

Visser, W., van der Baan, A. A., Batenburg-van der Vegte, W., Scheffers, W. A., Kramer, R. \& van Dijken, J. P. (1994). Involvement of mitochondria in the assimilatory metabolism of anaerobic Saccharomyces cerevisiae cultures. Microbiology 140, 3039-3046.

Visser, W., van Spronsen, E. A., Nanninga, N., Pronk, J. T., Gijs Kuenen, J. \& van Dijken, J. P. (1995). Effects of growth conditions on mitochondrial morphology in Saccharomyces cerevisiae. Antonie Van Leeuwenhoek 67, 243-253.

Washburn, M. P., Ulaszek, R., Deciu, C., Schieltz, D. M. \& Yates, J. R., III (2002). Analysis of quantitative proteomic data generated via multidimensional protein identification technology. Anal Chem 74, 1650-1657.

Washburn, M. P., Koller, A., Oshiro, G., Ulaszek, R. R., Plouffe, D., Deciu, C., Winzeler, E. \& Yates, J. R., III (2003a). Protein pathway and complex clustering of correlated mRNA and protein expression analyses in Saccharomyces cerevisiae. Proc Natl Acad Sci U S A 100, 3107-3112.

Washburn, M. P., Ulaszek, R. R. \& Yates, J. R., III (2003b). Reproducibility of quantitative proteomic analyses of complex biological mixtures by multidimensional protein identification technology. Anal Chem 75, 5054-5061.

Edited by: M. Molina 